\title{
De l'image au social : le chemin d'une évolution pour les pratiques en communication interne
}

\author{
Vincent Brulois, directeur de l'UFR Communication, \\ Université Paris 13, PRES Sorbonne Paris Cité, LabSIC, \\ brulois@sic.univ-paris13.fr \\ Jean-Marie Charpentier, responsable de \\ l'observation sociale d'une entreprise publique, \\ chercheur associé au LabSIC, France \\ cojm@club-internet.fr
}

L'entreprise est aux prises avec des complexités organisationnelles, sociales et territoriales de plus en plus fortes qu'une conception de la communication, indexée sur l'image, ne traduit pas. En première ligne, les communicateurs perçoivent la nécessité de s'extraire du cloisonnement par fonction pour jeter des ponts vers une conception plus sociale de la communication. Des frémissements sont perceptibles qui témoignent d'un questionnement et de possibles reconfigurations de l'approche de la communication dans les entreprises. C'est ce que nous ont dit, à leur manière, un certain nombre de directeurs de la communication interrogés depuis octobre 2009. De leurs propos, nous avons tiré des constats (sur la compréhension, sur la juste distance, sur la relation, sur le sens, sur les connaissances nécessaires) qui sont révélateurs d'une recherche d'articulation entre les individus, d'un souci de mettre à distance une approche réductrice de la communication au profit d'une communication au cœur du système social, au cœur du travail. 
Depuis janvier $2009^{37}$, 1'Association française de communication interne (AFCI) a engagé une réflexion sur les savoirs nécessaires aux pratiques professionnelles des communicants (version française) ou communicateurs (version québécoise). Constatant que la grande entreprise est aux prises avec des complexités organisationnelles, sociales et territoriales de plus en plus fortes, des adhérents ont perçu la nécessité de s'extraire du cloisonnement classique par fonction pour jeter des ponts vers une conception de la communication qui prenne en compte le fait que l'entreprise est d'abord «sociale» (Thuderoz, 1997, p.113). Rappel essentiel qui prend le contre-pied de la promotion d'un modèle communicationnel et gestionnaire univoque, tenant bien peu compte des individus, et reposant sur le développement et l'interconnexion (relative) des technologies de communication. Remettant en cause une certaine uniformisation des conventions de communication, les communicants ne cherchent-ils pas en fin de compte à redéfinir leur travail? Au-delà d'un rôle classique de traduction et de transmission des informations à différents publics, dans quelle mesure sont-ils tentés d'aller voir aussi du côté de l'analyse et de la compréhension de la diversité des individus, de la complexité de leurs relations ?

Des frémissements sont perceptibles, témoignant d'un questionnement et de possibles reconfigurations de l'approche de la communication dans les entreprises. C'est ce que nous ont dit, à leur manière, un certain nombre de directeurs de la communication ou des ressources humaines (RH) interrogés depuis octobre $2009^{38}$. De leurs propos, obtenus par entretien semi-directif ${ }^{39}$, nous avons tiré des constats qui révèlent à tout le moins un écart avec une conception de la communication indexée sur l'image - loin de toute dimension sociale - qui prévaut encore largement dans les entreprises. Chaque jour, de nombreux praticiens vérifient les limites d'une communication instrumentale à base de plans de com', d'outils, de

\footnotetext{
${ }^{37}$ Cet article fait suite à un premier, écrit il y a deux ans pour le 77e Congrès de l'ACFAS, dans lequel nous avons présenté les premiers pas d'une réflexion en cours (Brulois et Charpentier, 2009). Notamment, nous y relations le développement de l'AFCI et de sa volonté de participer activement à une réflexion critique sur la communication interne.

${ }^{38}$ Une vingtaine d'entretiens ont été conduits d'octobre 2009 à mars 2010 auprès de directeurs(trices) de la communication, de la communication interne (principalement) ou des ressources humaines (complémentairement) de grandes entreprises. Pour une grande majorité, ils n'étaient pas membres de l'AFCI. Nous avons donc bénéficié de la force du réseau de l'AFCI (afin d'avoir accès à ces responsables) tout en essayant d'en rester à distance (afin de voir si les thématiques portées par l'AFCI étaient aussi celles d'autres communicants).

${ }^{39}$ L'entretien se déroulait classiquement en deux temps. D'abord un rappel de l'objet de l'entretien (dans un contexte où les demandes des salariés vis-à-vis de leur entreprise augmentent et se font de plus en plus pressantes, quelle position peut adopter le communicant?). Ensuite l'entretien luimême structuré autour de cinq thèmes : sa formation et son parcours professionnel, l'évolution de son contexte de travail (changements et innovations), son métier et les compétences nécessaires pour l'exercer, le rapport entre la communication et les RH dans son entreprise, et enfin le lien de son entreprise avec les SHS (recherches, interventions, études) et son regard personnel sur les SHS (intérêt, utilisation).
} 
réseaux numériques et autres dispositifs venant buter sur la question sociale, mais relayant inlassablement un modèle communicationnel univoque. Face à ces limites et à la défiance de plus en plus grande vis-à-vis de la parole portée par l'entreprise à l'externe comme à l'interne, certains projettent déjà une nouvelle approche, un nouvel âge de la communication qui deviendrait «transformative (Habib, 2011). Force est de constater que les propos recueillis et les attentes qu'ils expriment apparaissent loin de cette «nouvelle » approche dont la caractéristique première est d'être un énième succédané de la communication «corporate » des années 1990, toujours aussi éloignée des dynamiques sociales.

Les propos que nous avons recueillis sont révélateurs d'une recherche d'articulation entre les individus, d'un souci de mettre à distance une approche réductrice de la communication au profit d'une communication au cœur du système organisationnel et du système social, en un mot au cœur du travail. Ils sont les indices d'une réflexion critique de ceux dont le travail est de communiquer, constituant une certaine forme de résistance aux normes de communication en vigueur. S'ils ne redéfinissent pas encore ces normes qui dictent leurs pratiques professionnelles, ils bousculent pourtant leurs certitudes et leurs habitudes de travail. En ce sens, le fameux adage énoncé par Paul Watzlawick est plus vrai que jamais : "On ne peut pas ne pas communiquer". Pourtant, s'agissant de la communication d'entreprise, tout porte à croire que la communication d'individu à individu est aujourd'hui difficile alors même que les réseaux numériques promettent des échanges démocratiques car horizontaux. C'est pourquoi nous nous sentons quelques affinités avec une sociologie centrée sur l'individu qui cherche à mettre en évidence le rapport à l'autre dans le cadre du travail, le rapport à l'entreprise, le rapport à la sociétét ${ }^{40}$.

\section{L'essoufflement du prêt-à-penser managérial}

De nombreuses voix se font l'écho d'une transformation du travail marquée entre autres par une profonde crise du management, ou plus exactement du système de représentations managériales accumulées et véhiculées à travers formations, livres, manuels et outils depuis plusieurs décennies ${ }^{41}$. Hier encore, un prêt-à-penser du management s'appuyant sur un vaste corpus international "aux confins de la gestion, de l'économie, de la sociologie et de la psychologie » permettait d'organiser

\footnotetext{
${ }^{40}$ Nous pensons principalement à Robert Castel (La Montée des incertitudes, 2009 ; Propriété privée, propriété sociale, propriété de soi, 2001) et Isabelle Ferreras (2007). De façon complémentaire, nous pouvons également citer Alain Ehrenberg (Le Culte de la performance, 1991 ; L'Individu incertain, 1995) et Richard Senett (Le Travail sans qualité, 2000).

${ }^{41}$ Avec Luc Boltanski, nous pouvons remonter jusqu'aux années 50 (Les cadres. La formation d'un groupe social, Minuit, Paris, 1982).
} 
l'entreprise selon des schémas, des outils et des méthodes portés par de grands cabinets de conseil (Segrestin, 2003, p.33). Au fil du temps, cette pensée - tenant parfois du mythe - accompagnée d'artefacts (gestion des processus, reporting ou key performance indicators) - tout à fait arrimés quant à eux au réel - ont fini par produire une doxa qui a servi de viatique à toutes les fonctions de l'entreprise et ont "charrié toutes sortes de représentations métaphoriques de l'entreprise qui apparaissent rétrospectivement comme autant de regards portés sur le monde » (ib., p.35). Entre croyances et applications, les techniques de management se sont ainsi déployé comme une «technologie politique», pour reprendre une expression empruntée à Michel Foucault (MispelblomBeyer, 2007, p.88), à l'initiative de directions d'entreprise qui, cherchant à remobiliser leurs équipes face à une concurrence mondialisée et à un marché moins favorable, ont "surorganisé le travail » en produisant règles, procédures et finalement confusion (Dupuy, 2010, p.7). À travers procédures et processus, la méfiance et le contrôle des risques ont été érigés en règle là où il fallait favoriser le goût du risque et la confiance dans l'échange et le travail en équipe. L'innovation en a sérieusement pâti tout autant que le "vivre ensemble"... Tant et si bien que la pratique managériale a pu être perçue comme «bro[yant] la singularité des êtres » (Fleury, 2011, p.23), obligeant chaque jour le salarié - manager de proximité y compris - «à suivre des préceptes qu'il désavoue intérieurement » (ib., 2010, p.62).

Dans ce cadre, le métier est redevenu une "ligne de défense » face au cours des choses (Rozès, 2011, p.18).Tout se passe comme s'il permettait aux individus de se concentrer sur un élément de stabilité alors que tout bouge autour d'eux déstructurant les collectifs de travail et leurs solidarités, comme s'il permettait de réexplorer leur utilité sociale dans un contexte de changement. Signe d'un repli sur soi et d'une frilosité face à l'avenir ? Peut-être... Plus certainement, à notre sens, réaction face à une vision managériale très simpliste. "Combien de dirigeants", s'interroge ainsi Marc Uhalde, «justifient leurs réformes par de pseudo théories qui relèvent plutôt de simples croyances comme la nécessité de la "rupture", la résistance "naturelle" des individus au changement ou encore la mutation du corps social par injection de "sang neuf"? » (2010, p.30). Cette vision ne prend pas la mesure de ce à quoi est confronté l'individu au travail, salarié comme manager de proximité. Surtout, elle occulte le fait que le «changement est une relation, un échange, et non la simple mutation de l'un des protagonistes» (ib., p.31). Bref, le changement est une construction sociale qui implique discussion, dialogue, débat en un mot, communication - afin de trouver un compromis pour un nouvel accord qui réinvente un équilibre, une régulation entre les individus. 
Dès lors, les salariés sont dans l'attente; dans l'attente d'échanges et de relations avec leur manager sur le travail et la façon de bien faire leur travail ${ }^{42}$. Cette attente est partagée par le manager de proximité. Mais, dans les faits, il ne peut y donner suite, saturé qu'il est par la multiplication des contraintes, notamment ce reporting qui l'oblige à continuellement se justifier du pourquoi et du comment, ou encore le transfert de tâches administratives ou de gestion RH qui doit faire de lui le «premier responsable RH de [son] équipe» (Peretti, 2006, p.21). De fait, le manager est le plus souvent «derrière son ordinateur que sur le terrain », alors même qu'il devrait «écouter, reformuler, expliquer» (Basilien et al., 2010, p.21)... Au même titre que les salariés de son équipe, le manager ne parvient plus à défendre les valeurs qui comptent pour lui, mis sous tension par des indicateurs de performance souvent contradictoires. Il peut être alors tenté de se rebeller ${ }^{43}$ pour faire son travail comme il l'entend! Situation paradoxale, mais surtout dangereuse pour l'entreprise, car si le cadre entre en rébellion, "il sort de son rôle traditionnel de "courroie de transmission", de "relais"» et passe de la confiance à la défiance vis-à-vis de sa propre hiérarchie (Courpasson, 2011, p.11). Ce qui se fait jour, c'est une «rupture dans la ligne managériale » entre la grande majorité de cadres - y compris supérieurs - et les quelques dirigeants qui prennent véritablement les décisions. Les stratégies sont de moins en moins partagées dans leur élaboration, renvoyant les premiers à l' « exécution de décisions auxquelles ils [n'ont] pas participé» (Basilien et al., 2010, p.20). Le management de proximité est mis en porte à faux, renforçant la crise d'un certain modèle managérial et laissant l'entreprise en passe de "perdre le contrôle d'elle-même » (Dupuy, 2011).

Assurément, des craquements se font sentir et la crise financière commencée à l'été 2008 n'a pas fini de produire son onde de choc économique et sociale. Longtemps prônée, l'invitation à la reproduction du modèle managérial est désormais considérée, par ses propres auteurs, comme contre-productive. Les uns clament ainsi que le capitalisme est undersiege, signant ainsi un constat d'échec des modèles de gestion passés qu'ils ont contribué à confectionner et d'une «outdated approach » de ce que doit être la création de valeur ${ }^{44}$ (Porter et Kramer, 2011, p.4). Le questionnement porte moins sur ce qui a conduit à cette situation que sur ce qu'il convient de changer dans le modèle afin de refonder la légitimité du capitalisme ${ }^{45}$. À

\footnotetext{
${ }^{42}$ Voir notamment Yves Clot: Le Travail à cœur. Pour en finir avec les risques psychosociaux (La Découverte, Paris, 2010).

${ }^{43}$ Selon le titre du livre de David Courpasson et Jean-Claude Thœnig, Quand les Cadres se rebellent (Vuibert, Paris, 2008).

${ }^{44}$ Selon ces auteurs, «the concept of shared value can be defined as policies and operating practices that enhance the competitiveness of a company while simultaneously advancing the economic and social conditions in the communities in which it operates » (Porter et Kramer, 2011, p.6).

${ }^{45}$ Porter et Kramer insistent en précisant: « The purpose of the corporation must bere defined as creating shared value, not just profit per se. » (2011).
} 
cette occasion, on s'interroge sur les référents des formations, en tout cas ceux qui sont enseignés depuis des décennies dans les écoles de management. Une étude récente $^{46}$ montre ainsi que, au reproche classique d'une formation «trop peu critique ", s'ajoute celui d'une formation «trop éloigné[e] de la vie réelle» (Hatchuel, 2011)! Diplômés d'un MBA, des apprentis dirigeants se sentent même insuffisamment formés au moins dans trois situations :

1. lorsqu'il s'agit de changer d'échelle et de diriger non plus un service - qu'ils peuvent toujours «entraîner par l'exemple» - mais une direction - où il s'agit d'« être le garant d'un collectif efficace et juste »;

2. lorsqu'il s'agit, non plus de répéter des règles ou des processus appris par ailleurs et dont ils ne questionnent plus le bon usage, mais d'" apprendre de nouvelles méthodes », d'acquérir de nouvelles compétences et d'adopter une autre réflexion pour inventer de nouveaux modes d'action ;

3. lorsqu'ils se trouvent face à des dilemmes éthiques, de plus en plus nombreux, et qu'il s'agit de "défendre leur position», de débattre, de discuter afin de sortir du consensus et de «trouver un compromis » (idem).

Nombre de ces formations ont pourtant construit leur réputation sur la promesse d'acquérir des compétences mobilisables en situation professionnelle qu'ils assimilent facilement, au passage, à des savoirs universitaires. Cette «transposition pragmatique » opère comme prescription d'aptitudes rédigées le plus souvent sous la forme de propositions infinitives, lesquelles ne requièrent le verbe savoir qu'en sa qualité d'opérateur modal : savoir gérer, savoir décider, etc. (de la Broise et Brulois, 2010, p.125). Écrivant la norme du fait de leur position dominante, ces formations se retrouvent pourtant interpellées sur leur incapacité à former leurs étudiants à comprendre, à donner du sens et à agir en trouvant la bonne distance face aux êtres et aux choses de l'existence. C'est pourquoi Henry Mintzberg insiste sur le fait de se concentrer sur l'«apprentissage des méthodes de réflexion » au lieu de "prétendre enseigner le management», proposant même que ces formations abandonnent $1^{1}$ '«enseignement des fonctions - c'est une perte de temps - et [se remettent] à la sociologie, à la philosophie, à l'histoire, à l'anthropologie, etc. » (2009). Les propos avaient des allures de mea culpa collectif. D'une certaine façon, ces spécialistes reconnaissaient que le roi était nu et que la doxa longtemps diffusée était en cause.

\footnotetext{
${ }^{46}$ Étude menée par Beth Benjamin et Charles O.Reilly (« Becoming a Leader : EarlyCareer Challenges Faced by MBA Graduates », Academy of Management Learning and Education, vol.10 n³, septembre 2011) et citée par (Hatchuel, 2011).
} 


\section{Le communicant face à de nouvelles interrogations}

La communication n'a pas échappé à ce mouvement de rationalisation commandé par une certaine approche managériale. À bien des égards, ses praticiens sont au cœur de dilemmes nouveaux et leurs missions sont questionnées par le mouvement même des entreprises. Ils ont longtemps privilégié - et privilégient encore pour certains - une dimension utilitariste et technique de la communication d'entreprise sans s'interroger sur le sens de celle-ci, notamment la question des savoirs permettant de comprendre les transformations de l'entreprise. Mais les entretiens que nous avons menés nous montrent que des déplacements sont en cours. Les communicants sont en tension, s'appuyant sur une opinion diffuse - une doxa - tout en cherchant plus ou moins explicitement dans le contexte troublé d'aujourd'hui un savoir - une épistèmê. Cette tension est un symptôme parmi d'autres de la crise du logiciel managérial en tant qu'outil polyvalent pour raconter le monde de l'entreprise et agir sur lui.

C'est dans ce contexte que l'AFCI organisait en mars 2007 un séminaire sur l'apport des sciences humaines et sociales (SHS) à la communication. Une interpellation propre à l'association a suivi, rappelant le rôle premier de la communication, à savoir la relation à l'autre bien avant tous les plans, outils ou autres dispositifs : «Quels sont nos fondamentaux scientifiques, et surtout comment assurons-nous le lien permanent entre le matériel analytique et conceptuel qu'ils nous proposent et les pratiques que nous développons? 》(AFCI, 2008, p.1). Cette interpellation a suffisamment porté pour qu'un groupe de travail, réunissant praticiens, professionnels d'agence, chercheurs, soit constitué en janvier 2009. Dès le départ, la vocation de cet atelier a été à la fois de développer des réflexions et de mettre en place des événements utiles à toute l'association. C'est dans ce sens qu'une enquête par entretiens a été initiée afin de comprendre la place et le rôle de la communication dans l'entreprise, son évolution et d'analyser la nature du rapport de ces praticiens avec les SHS dans leur activité ${ }^{47}$. Parallèlement, une formation sur l'apport de ces sciences à la communication a été mise en place proposant aux adhérents des éclairages théoriques sur les transformations des organisations et leur contexte sociall $^{48}$. Enfin, le travail de l'atelier a permis à l'AFCI de nouer des liens ${ }^{49}$ et de tisser des coopérations ${ }^{50}$.

\footnotetext{
${ }^{47}$ Une restitution de ces entretiens a été réalisée sous la forme d'une conférence-débat entre professionnels et universitaires le 6 juillet 2010 au siège d'AXA.

${ }^{48}$ La formation a été organisée autour des apports de la sociologie (Norbert Alter, Marc Uhalde, Florence Osty, Philippe Zarifian), de la psychosociologie (Florence Giust-Desprairies), des SIC (Vincent Brulois), de l'ergonomie (François Hubault) et des sciences de gestion (Maurice Thévenet, Étienne Normand).
} 
Ces initiatives témoignent d'un souci réactualisé de l'AFCI de mettre à distance l'approche instrumentale de la communication, dont les limites sont de plus en plus manifestes, au profit d'une communication au cœur du système organisationnel et social. Depuis 1989, l'activité de l'AFCI est émaillée d'une volonté de concevoir la communication interne comme un «territoire partagé entre les fonctions et entre les individus » au lieu d'en faire un territoire réservé aux seuls communicants, de se construire comme une structure de réflexion et de prospective, c'est-à-dire d'être à la fois dans l'action et dans la réflexion (Brulois et Charpentier, 2009). Surtout, il y a l'idée qu'on ne peut pas penser la communication aujourd'hui en dehors des connaissances qui concernent tant le rapport de l'entreprise à la société que les systèmes sociaux internes, le sociétal et le social. L'intérêt marqué pour les SHS de la part d'une association qui compte dans la profession ${ }^{\mathbf{5 1}}$ constitue en soi un indice qu'un mouvement est en cours dans ces métiers confrontés à une grande transformation des entreprises.

D’une certaine façon, mondialisation aidant, ces dernières ont développé leur omniprésence - être présent partout et tout le temps par une communication médiatisée - favorisant ainsi une rationalisation des pratiques de travail, mais aussi une distance de plus en plus importante "entre les enjeux vus d'une direction générale et la réalité des préoccupations d'un salarié dans son environnement professionnel et personnel » (Basilien et al., 2010, p. 20). Les options stratégiques ne font plus l'objet d'un débat a minima, la stratégie devient illisible pour les salariés comme pour les managers, affaiblissant considérablement l'adhésion de tous à la stratégie finalement choisie. Il est alors moins paradoxal qu'il n'y paraît de constater un mécontentement grandissant des salariés vis-à-vis des stratégies menées par leur entreprise «à un moment où l'on n'a jamais autant communiqué sur celles$c i »(i b .$, p.19) ! Cette insatisfaction renvoie aux non-dits des stratégies annoncées (par exemple, quant aux autres options possibles...) qui favorisent le sentiment que «les plans véritables sont cachés aux salariés » (ib., p.20). À trop oublier le local et le social, ils se rappellent aux entreprises et à leurs dirigeants de manière ponctuelle,

\footnotetext{
${ }^{49}$ Avec Entreprise \& Personnel, un réseau associatif créé en 1969 consacré à la gestion des RH et au management des hommes dans les organisations. Ce réseau regroupe aujourd'hui plus de 110 entreprises et autres institutions et a un rôle de veille, de publication, d'expertise et de formation.

${ }^{50}$ Avec l'Association des professionnels en sociologie de l'entreprise, créée en 1998 autour de Renaud Sainsaulieu, en même temps que la revue Sociologies Pratiques qui lui est liée. Ou encore avec la Société française des sciences de l'information et de la communication (SFSIC), créée en 1974 comme société savante des chercheurs de la discipline.

${ }^{51}$ Il existe bien en France d'autres associations regroupant des communicants au sens large. Mais seule l'AFCI s'intéresse spécifiquement à la communication interne. Cela ne signifie pas pour autant que tous les communicants travaillant à l'interne sont membres de l'AFCI...
} 
mais radicale, tout au moins en France ${ }^{52} \ldots$ Une façon pour les salariés de dire que l'omniprésence communicationnelle ne peut être que virtuelle et ne remplace pas le besoin de co-présence - être ensemble ici et maintenant - indispensable pour créer de la cohésion sociale.

\section{Comprendre pour mieux agir}

Partir à la recherche d'un social perdu. C'est en somme l'objet que se sont donné les participants de l'atelier de l'AFCI, c'est avec cette ambition que nous sommes allés à la rencontre de praticiens. Les entretiens réalisés, que nous complèterons par les résultats d'une enquête quantitative menée à la même époque ${ }^{53}$, font apparaître cinq constats qui montrent au moins autant les tensions que vivent les praticiens que les attentes dans un moment de transformation majeur.

Premier constat, le besoin de compréhension a rarement été aussi fort. Les communicants sont souvent «dans la production de supports 》 et « ils restent dans la forme et ont du mal à comprendre les enjeux internes $»{ }^{54}$. Mais, aujourd'hui, ils ne peuvent plus se cantonner aux outils et aux seules compétences techniques, car le défi est de "comprendre le milieu» dans lequel ils interviennent, «comprendre comment on peut mettre en mouvement des enracinements collectifs » en interne et viser la «compréhension stratégique des enjeux » de l'entreprise dans un contexte d'incertitude et de déséquilibre. Plus que jamais, ils doivent être à l'écoute du corps $\operatorname{social}^{55}$, à l'écoute des «signaux faibles » afin de pousser à une compréhension plus fine, au-delà du superficiel, de relier les niveaux (du micro au macro) et de multiplier les regards. En une formule, ils doivent être les «cinq sens de l'entreprise ", tout à la fois dans l'écoute, la perception, le ressenti et, in fine, dans la compréhension. Leur objectif est d'acquérir une vraie place stratégique dans

\footnotetext{
${ }^{52}$ En septembre 2010, une enquête TNS-SOFRES-Publicis Consultants indiquaient que $87 \%$ des salariés se disent« convaincus que les intérêts des dirigeants et des salariés ne vont pas en France dans le même sens » (repris dans Basilien et alii, 2010, p.36)!

${ }^{53}$ Il s'agit du baromètre de la fonction Communication interne réalisé par le cabinet Inergie (qui accueille dans ses locaux les bureaux de l'AFCI) pour le compte de l'AFCI en octobre 2009. Ce baromètre a été établi à partir d'une enquête approfondie par questionnaire administrée aux membres de l'AFCI : 278 réponses de la part de chargé ou responsable de communication travaillant en grande entreprise ou en entreprise de taille intermédiaire. Ces données de seconde main sont intéressantes en ce qu'elles élargissent le spectre du positionnement hiérarchique du communicant (des chargés ou responsables alors que nous avons interviewé des directeurs) tout en renforçant nos données de première main.

${ }^{54}$ Sauf indication contraire, ce verbatim comme les suivants est issu des entretiens menés.

${ }^{55}$ Développer l'écoute du corps social est un des trois items en forte hausse dans le baromètre de la fonction Communication interne (Inergie, 2009, p.18).
} 
l'entreprise alors même que le contexte se durcit ${ }^{56}$. Cette envergure stratégique est jugée d'autant plus primordiale que son absence cantonne leur activité à de la production de marque, d'image, de visibilité, autrement dit, à une production qui méconnaît «la structure, l'organisation, la culture, les valeurs de l'entreprise et des hommes».

Deuxième constat, le communicant doit « se situer » et a besoin de distance. Il doit apprendre à «porter un regard critique, à prendre de la distance et du recul par rapport aux événements». Il a vocation à devenir un expert stratégique qui doit «être à la fois dans et à distance de l'entreprise». Une posture à présent nécessaire... même si elle est loin d'être aisée, car « elle le place en situation de critiquer une stratégie et de pointer les faiblesses de l'organisation ». C'est donc à un «rôle d'entre-deux» qu'il est convié. Il lui faut être «impliqué, mais détaché », il lui faut être à la fois un «technicien» (maîtriser les outils), un "écrivain ou plus exactement un "nègre" " (écrire, mettre en mots) et un "rebelle vis-à-vis de sa direction générale» (critiquer). Cette mission relève souvent de la gageure et requiert à tout le moins une compréhension des jeux et des enjeux des acteurs, un positionnement en équilibre entre direction, managers et salariés. L'intérêt point déjà d'un recours aux SHS, afin de prendre de la distance par rapport aux outils, de se recentrer sur l'individu, d'arriver à une meilleure compréhension du corps social ${ }^{57}$.

Troisième constat, le communicant est au cœur des relations dans un rôle d'intermédiation sociale. L'intelligence nécessaire de la situation lui donne les moyens d'être un facilitateur de relation entre le haut et le bas de la hiérarchie, comme de façon transversale entre les métiers. Le rôle de la communication interne est alors de "développer de la relation, de la coopération entre les individus, de l'engagement $»$. Il s'agit de relier les individus ${ }^{58}$ plutôt que de relayer l'information. Relayer signifie que l'émetteur est le seul pourvoyeur de sens, sens qu'il s'agit de transmettre à des cibles, autrement dit des inconnus sans nom et sans visage! L'accent est donc mis sur les outils. À l'inverse, relier signifie que communiquer c'est mettre en commun et que l'accent est mis sur la nature et la qualité des relations: "aujourd'hui, c'est moins l'émetteur et le récepteur qui comptent que le contenu et la qualité de la relation établie ou rétablie quand il y a eu tension ou conflit». C'est bien cette seconde voie qui est privilégiée par une majorité de praticiens pour que la communication interne soit la fonction qui remet l'individu au centre, pour que le communicant devienne un « responsable des relations internes ».

\footnotetext{
${ }^{56}$ Perte de repères des salariés, éloignement des décisions, individualisation du travail, problèmes de cohésion sociale (Inergie, 2009, p.34).

${ }^{57} 56 \%$ des responsables de communication interne disent avoir recours aux SHS dans leur pratique professionnelle, principalement pour les raisons citées (Inergie, 2009, p.22).

${ }^{58}$ Favoriser les coopérations et décloisonner est un des trois items en forte hausse dans le baromètre de la fonction Communication interne (Inergie, 2009, p.18).
} 
L'idée sous-jacente est que les individus peuvent - doivent - être des acteurs et non de simples récepteurs. Qui plus est, à l'heure du Web 2.0, l'action des communicants à moins à voir avec «un pouvoir normatif de contrôle de l'information » qu'avec « un pouvoir d'accompagnement ${ }^{59}$, d'ouverture, de mise en relation ». Ils doivent réagir vite, de plus en plus vite, tout en intégrant que les processus de changement restent, quant à eux, inscrits dans le temps long.

Quatrième constat, le communicant est au cœur de la production et de la transmission de sens. Dans son rôle de «traducteur, vulgarisateur, simplificateur », il est un passeur de sens, car la mission de la communication interne est de «trouver l'équilibre entre ce qui fait sens pour le personnel et ce qui fait sens pour la direction générale » dans un contexte d'urgence permanente. Le rapport au temps est encore (toujours) présent. Dans cette phase de grande transformation $^{60}$ des entreprises, il importe en interne comme en externe de faire comprendre où l'on va : «Plus on se transforme, plus on évolue; plus la compréhension du sens, des contraintes et des finalités deviennent essentielles»! Cette compréhension se décline à deux niveaux. Pour l'entreprise, il s'agit d'éviter le grand écart entre le construit stratégique et la réalité ${ }^{61}$, car cette distorsion est mortifère, favorisant le désengagement des individus, leur défiance quant à la parole de l'entreprise. Pour les individus, cette question renvoie à leur place, à leur rôle dans l'organisation, à leur utilité, à la reconnaissance qu'ils attendent en retour, à leur identité pour euxmêmes comme pour autrui. Il est donc important de traduire les messages et de transmettre un sens collectif, car aujourd'hui, il s'agit moins d'améliorer les conditions de travail (même si cela constitue encore une évidence dans certains secteurs, pour certains métiers), que de maintenir le sens même du travail. Et comment ne pas éviter la perte de sens si, dans leur quotidien, «là où les enjeux sont les plus vifs et les mieux compris» (c'est-à-dire au travail), les salariés et cependant toujours citoyens «sont de fait traités comme des domestiques, et ce, malgré la meilleure des "gestions de ressources humaines" (Ferreras, 2007, p.251) ?

Cinquième constat, un socle de connaissances devient indispensable. Les praticiens appellent en quelque sorte à un nouvel équilibre entre compétences techniques - déjà présentes, toujours utiles dans la panoplie du communicant mais pas suffisantes - et capacités analytiques jusqu'alors sous-estimées mais seules susceptibles de capter des éléments de connaissance du corps social, de les analyser

\footnotetext{
${ }^{59}$ Le baromètre note une percée certaine mais encore balbutiante des espaces collaboratifs. Il note aussi que leur déploiement ne peut se passer d'un accompagnement jugé important et revendiqué par les communicants internes (Inergie, 2009, p.21).

${ }^{60}$ Selon le titre du livre de Karl Polanyi : La Grande transformation : aux origines politiques et économiques de notre temps, Gallimard, Paris, 1983 (1e éd. : 1944).

${ }^{61}$ Crise aidant, les actions de communication interne sont devenues régulières et orientées vers la pédagogie : il faut expliquer les enjeux, donner du sens. Mais les praticiens regrettent le manque de proximité et le peu d'adéquation avec les publics auxquels elles s'adressent (Inergie, 2009, p.25).
} 
et de les utiliser pour agir. Or, ces capacités analytiques puisent à la source des SHS, dont l'utilisation apparaît comme un nouvel enjeu pour les communicants ${ }^{62}$. Tous ne s'y réfèrent pas spontanément ni explicitement. Certains évoquent quelque chose qui relève de l'ordre de la connaissance. D'autres parlent très directement des SHS : "J'ai découvert que les SHS étaient des clés de compréhension de phénomènes qu'on ne peut pas comprendre autrement». La majorité comprend en tout cas qu'elles proposent des clés de compréhension - avant les clés d'action - pour des sujets dont ils ont de plus en plus à s'occuper: crise bien sûr, développement durable toujours, mais aussi questions $\mathrm{RH}^{63}$. In fine, elles apportent « $d u$ fond et de l'ancrage » pour comprendre les enjeux, elles apportent «de la stabilité, c'est ce qui assouplit la contrainte ». Bref, «c'est ce à quoi l'on se rapporte quand tout part à vau-l'eau!»

De ces entretiens et de cette enquête, il apparaît que la communication interne est aujourd'hui « ouverte sur ses partenaires internes » (RH, communication externe), «plus proche de ses relais» (managers) et «ouverte aux SHS» (Inergie, 2009, p.36). De façon paradoxale, la crise a ainsi produit un double effet sur elle: réduisant les budgets qui lui sont attribués, mais renforçant sa légitimité. Face au désarroi des salariés, les communicants tentent de se repositionner et de «se réapproprier les questions liées au travail, discuter du sens du travail, du temps de travail, des relations, de la reconnaissance de l'organisation " (Basilien et al., 2010, p.27).

\section{D'une communication faussement transformative à une communication dialogique}

Face à un tel objectif, on comprend que les communicants aient bien besoin de ce quelque chose de l'ordre des SHS, même si "ce prisme est encore peu partagé » dans l'entreprise. Toutefois, cette référence explicite à des connaissances est loin de résoudre le problème insoluble de la juxtaposition des temps auquel ils sont confrontés $^{64}$ : "Ce que j'apporte comme matériau de compréhension ne peut se traiter que par des actions de long terme car on est dans plusieurs décennies de moulage identitaire. Cela exige du temps. Or, les hommes d'action veulent la

\footnotetext{
${ }^{62}$ L'utilisation des apports des SHS (20\% des réponses) apparaît pour la première fois comme une évolution souhaitable de la communication interne (Inergie, 2009, p.33).

${ }^{63}$ Telles que réductions d'effectifs, évolution des métiers, vieillissement de la population, emploi des seniors (Inergie, 2009, p.32).

${ }^{64}$ L'agenda des communicants se répartit entre du temps consacré à l'animation de réseau (très peu), à l'animation d'équipe (peu), au relationnel interne (pas assez à leur goût : $25 \%$ de leur temps contre un tiers souhaité), à la conception et à la gestion du système d'information (trop à leur goût : $45 \%$ de leur temps contre un tiers souhaité) (Inergie, 2009, p.14).
} 
décision qui va donner des résultats la semaine prochaine! „ Entre temps court et temps long, la difficulté reste ainsi de conjuguer trois rythmes : celui de l'action, celui de l'adaptation de l'individu aux changements, celui de la réflexion, de l'analyse, de la mise à distance. Il ne fait plus de doute que dire le temps devient un enjeu stratégique majeur; et ce d'autant plus que l'entreprise est passée de la mise en place d'une succession de changements finis à un «mouvement permanent qui mobilise l'ensemble des acteurs » (Alter, 2000). Rythmer, c'est alors désigner ce qui est continuité ou rupture dans ce mouvement permanent, c'est identifier les «liaisons longitudinales entre le passé, les cultures collectives, les compromis sociaux et leur avenir» (Uhalde, 2010, p.33). Cette «capacité à décoder »les collectifs ancrés dans une histoire et une culture, les communautés qui se font et se défont au gré des projets, les environnements qui se décloisonnent et se recoupent est, à leurs yeux, le sésame qui sortira le communicant et la communication interne du monde des illusions et de la belle image.

La partie est pourtant loin d'être gagnée. Des tentatives pour transformer la communication sont à l'œuvre. Partant du constat que l'entreprise et la société civile sont dans une situation d' « incompréhension radicale », Laurent Habib par exemple juge la communication « coupable d'avoir maquillé un décalage toujours plus grand entre économie réelle et économie virtuelle », " coupable d'avoir décrédibilisé l'autorité entrepreneuriale en transformant les grands patrons en figures médiatiques », « coupable d'avoir entretenu l'illusion d'un libéralisme vertueux et moral par nature » (2011, p.5). Personnifiée, la communication d'hier est donc seule condamnée (alors qu'elle était instrumentalisée) laissant le champ libre à une « communication transformative » qui aurait vocation à « donner une valeur pérenne au capital de richesse des marques, nourrir une relation durable avec les publics, rendre la transformation sociale possible et le changement humain » (ib., p.6). Bien loin d'en appeler à une communication sociale, il prône une nouvelle communication publicitaire qui permettra de créer de la valeur commerciale et financière. La marque «totale » (sic) sauverait ainsi l'entreprise (idem), redorerait son image défaillante et lui permettrait de redevenir une institution centrale de cette « higherform of capitalism » espérée par Michaël Porter (2011, p.15). Dans ce cadre, les communicants devraient apprendre à travailler autrement afin de « renforcer jour après jour le capital de valeur des marques » (Habib, 2011, p.7)... Image un jour, image toujours, fut-elle de marque ! Nous revient alors à l'esprit la célèbre formule de Tancredi dans Le Guépard : "Si nous voulons que tout reste tel que c'est, il faut que tout change » (di Lampedusa, 1958). La parole de l'entreprise connaît une défiance sans précédent aussi bien en interne qu'en externe, la communication spectacle est à l'agonie, et il ne semble pas que cette communication "transformative" puisse y remédier d'une quelconque façon...

Petit à petit, l'entreprise s'est constituée comme institution centrale de la société et elle est aujourd'hui reconnue comme telle. Mais elle est dorénavant immergée dans 
un rapport interne/externe plus politique: "l'entreprise, c'est compliqué, c'est violent et ça va vite»! Cette remarque fait mouche, car elle montre toute la complexité qui touche l'organisation de l'entreprise, toute la violence qui sourd des rapports sociaux et des relations individuelles de travail, toute la vitesse à laquelle se succèdent les changements, s'impulse le mouvement et fait bouger la culture, les identités, les métiers de l'entreprise. Et ce n'est pas - ce n'est plus - une politique de marque, fût-elle transformative, qui va résoudre l'équation de la communication d'entreprise !

La contrepartie de l'institutionnalisation est une demande de justice démocratique, singulièrement de la part des salariés, mais aussi d'autres acteurs dans la société. Hier, l'entreprise débordait la société en y introduisant ses références (organisation du temps, recherche de la performance, rapport aux autres). Aujourd'hui, à bien des égards, c'est la société qui déborde dans l'entreprise à travers des demandes sociales, environnementales et démocratiques - en bref politiques - de plus en plus pressantes de la part de salariés espérant être pleinement considérés comme des citoyens au travail. La porosité des environnements ne se vérifie pas seulement pour les entreprises, mais aussi pour les individus... Cette demande oblige pour le moins les entreprises à revoir en interne des «règles de la vie commune au travail» (Ferreras, 2007, p.119), à organiser des «dialogues critiques sur le terrain » (Hatchuel, 2010) et à modifier en externe leur politique de communication et leur rapport à la société ${ }^{65}$. Qu'elle le veuille ou non, l'entreprise doit donc faire face à l'externe comme à l'interne à des enjeux de caractère plus politique qu'une communication de marque n'est pas de nature à traiter. Seules des formes renouvelées de dialogue - social à l'interne et civil à l'externe - peuvent être à la hauteur des enjeux.

Deux observations au moins rendent la période favorable pour la communication interne. D'une part, l'accroissement de la taille des entreprises et leur activité mondialisée ont accru la distance entre décideurs et salariés. Une mise à distance des individus s'est opérée qui modifie de façon notable les comportements de ceux qui travaillent dans l'entreprise et plus encore de ceux qui la dirigent: «on n'assume pas ses responsabilités de la même manière si on est au contact immédiat de ceux qui en subissent les conséquences », remarque Pierre-Yves Gomez (2010)! Cet éloignement est dû notamment aux outils de management dont l'usage généralisé a favorisé les échanges médiatisés et indirects au détriment de rencontres en face à face et donc en temps réel. De fait, le reporting a remplacé le « raisonnement» déresponsabilisant les décideurs et les managers, sur-organisant le travail des salariés (Tixier, 2010, p.17). D'autre part, le recentrage de la fonction RH sur ses activités à valeur ajoutée s'est traduit par un mouvement d'externalisation (sous-

${ }^{65}$ Voir (Brulois et Viers, 2009). 
traitance voire délocalisation) et un mouvement de délégation (aux managers opérationnels). Tant et si bien que l'activité s'est alors technicisée (portail informatique, ERP pour Enterprise Resource Planning ou progiciel de gestion intégré en français), normalisée (des décisions prises au niveau mondial et déployées selon une rationalité unique), éclatée (l'activité RH se fait partout, même en dehors de l'entreprise), déshumanisée (plate-forme de services à distance). Là encore, force est de constater que la fonction RH s'est éloignée des individus - un comble! - du fait de la mise en place d'outils à la prétention universelle remplaçant l' " analyse de situations concrètes et [faisant] déni de la réalité» (idem). Une «vision désincarnée »s'est imposée, perdant ainsi la « compréhension en profondeur » des individus, de l'organisation et du travail (Le Gall, 2010). Le mal est fait et les RH apparaissent aujourd'hui affaiblies. Une succession de «dispositifs technicoéconomiques »- des ressources - ont éloigné les individus des relations qu'ils attendaient (Tixier, 2010, p.16). Du jour au lendemain, il est difficile de redonner un profil social à une fonction qui avait, hier encore, d'abord vocation à être un « business partner ». Les individus ne sont plus dupes, et même les salariés les plus dépourvus de ressources sont toujours autant attachés à la valeur Travail et en demande d'équité et de justice au sein de leur entreprise ${ }^{66}$.

Dans ce contexte, quel rôle la communication peut-elle tenir ? S'il semble clair que l'entreprise est « engagée » dans la société (Enlart, 2011), y a-t-il la place pour une communication qui fasse d'abord le pari des individus qui la composent, ceux-là mêmes qui font entrer la société dans l'entreprise?

Indéniablement, deux communications se font encore face! L'une, externe, qui cherche "à présenter l'entreprise sous un jour toujours positif», communication publicitaire renforçant la marque ; l'autre, interne, qui cherche à «faire connaître et comprendre les réalités souvent difficiles, les enjeux de l'entreprise, ses difficultés », communication plus sociale plongeant résolument dans les réalités des situations vécues par les salariés et dans leurs pratiques sociales (Basilien et al., 2010, p.39). Aujourd'hui comme hier, tout porte à croire que cela fait encore sens de parler de communication interne dans les entreprises et ses praticiens, s'ils savent s'appuyer sur leurs interrogations, sur leur quête de savoirs, sur une réflexion critique de l'organisation et de ses enjeux, peuvent devenir des acteurs incontournables et aider à penser de nouveaux modes de régulation en relation notamment avec les managers de proximité. Sans constituer une démarche critique, cette réflexion s'attache tout de même à dévoiler et à remettre en cause un ordre social, à en identifier les enjeux et les conséquences sur leurs pratiques professionnelles comme sur eux-mêmes en tant qu'individu.

\footnotetext{
${ }^{66}$ Voir à ce sujet le travail mené par Isabelle Ferreras sur les caissières de supermarché (2007).
} 
Le contexte actuel joue pour une telle évolution, car la maîtrise de la quantité d'information et le soin apporté à la qualité de l'information sont devenus deux défis majeurs des entreprises dans leur complexe rapport au temps et à l'action. Si la réponse à cette complexité est forcément technique, elle doit être «d'abord et fondamentalement humaine » (d'Almeida, 2009, p.15). Accélération du temps - qui semble «consacrer la pérennité du présent» - et surcharge d'informations - où les "événements s'accumulent mais ne font plus sens » (Augé, 2010) - sont deux atouts a contrario pour renforcer le dialogue interne seul susceptible de « recomposer un tissu social atomisé, gangrené par un sentiment d'urgence, voire d'impuissance » (d'Almeida, 2009, p.15). Il y a donc nécessité d'apporter sens, relation, proximité et conversation ici et maintenant ! Il y a nécessité à reconquérir le travail dans toutes ces dimensions, à la fois comme «facteur de production" (le travail comme gagnepain), comme «essence de l'homme» (le travail comme moyen de s'épanouir) et comme «support de droits et de protections» (le travail comme moyen de s'intégrer) (Méda, 2011). Il y a nécessité à rendre au travail toute l'«épaisseur sociologique qui l'anime » (Ferreras, 2007, p.16).

La communication peut y aider et être un des vecteurs pour sortir de cette crise du management si ses praticiens en entreprise savent la présenter, par leurs pratiques, comme ce qu'elle est ; une production aux multiples facettes, à la fois :

- "production symbolique », qui mobilise des langages, des normes, des valeurs configurant action et acteurs ;

- "production technique», aux formats multiples dont il faut aborder les « effets »avec humilité et sans fantasme (la question de ce que fait l'émetteur au récepteur et inversement) et dans sa complexité (la question de la coconstruction du message et de son sens);

- «production sociale », dans laquelle se façonnent des relations et des modes de sociabilité dans le cadre d'une organisation donnée; ces relations modifiant en retour l'organisation ;

- "production politique » enfin, dans laquelle se joue la dialectique incessante de la coopération et du conflit (d'Almeida, 2009, p.15).

Résolument inscrite dans une perspective stratégique qui fait large place aux individus (communication sociale), elle sera alors clairement démarquée d'une com' (communication publicitaire) qui ne cherche qu'à façonner une réputation immaculée à destination de cibles sans visage.

La réflexion critique menée par quelques communicants et portée par 1 'AFCI est assurément un exemple intéressant qui emprunte ce chemin. Il faut le suivre de près (et en identifier d'autres) même si de nombreuses questions restent en suspens. Par exemple, tout en participant activement à structurer la communication interne en France, l'AFCI n'est pas le seul acteur. Quels sont ces autres acteurs de la communication interne? Comment l'action de l'AFCI s'inscrit-elle dans les 
pratiques et réflexions menées par ces associations, fédérations ou syndicats professionnels? Quels sont les enjeux autour desquels se positionnent tous ces acteurs ? En outre, de quelle façon la réflexion critique actuelle se traduit-elle par une évolution du référentiel métier en communication interne? L'enjeu est d'importance pour la professionnalisation en communication interne. Alors que les mondes de la formation professionnelle et celui des RH se sont structurés et fortement professionnalisés, le monde de la communication - singulièrement interne - manque encore cruellement d'une structuration métier marquée. Cet élément structurant pourrait-il être l'individu, quitte à redécouvrir l'importance de la proximité, des relations, du social ? D'un monde à l'autre, dans quelle mesure les référentiels universitaires de formation en communication pourraient-ils infléchir les référentiels métiers au lieu de seulement s'y ajuster? Après tout, nous venons de voir que des praticiens questionnent leur métier et leurs compétences clés, se détachant de compétences mobilisables en situation professionnelle (des savoir-faire si ce n'est des savoir-être) pour rechercher des compétences plus analytiques, bien plus susceptibles selon eux de les aider à comprendre et, par suite, à agir. Quand bien même il s'agirait d'un jeu d'écriture, les référentiels de compétences interrogent bel et bien le processus de professionnalisation. Enfin, de façon plus générale, n’y a-t-il pas mesure à s'interroger sur les conditions nécessaires qui permettent à l'individu de conduire une réflexion sur ses propres pratiques ? Cela mérite certainement au moins une discussion, non seulement pour les communicants (en entreprise), mais aussi pour nous-mêmes enseignants et chercheurs, responsables de formation en communication.

\section{Bibliographie}

d'Almeida, N. (2009, décembre). L'omni-présent. Cahiers de la communication interne, (25), 14-15.

AFCI (2008, avril). Les sciences humaines n'intéressent-elles plus les communicants? Les Cahiers de la communication interne, (22).

Alter, N. (2000). L'Innovation ordinaire, Paris : éditions PUF.

Augé, M. (2010, 10 juillet). Réconcilier doute et espoir. Le Monde.

Basilien, J-P., Gilabert, M. et Monneuse, D. (2010, octobre). Découplages : le social sous contrainte, Note de conjoncture sociale d'Entreprise \& Personnel, 292.

de la Broise, P. et Brulois, V. (2010, automne). La communication interne aux prises avec la professionnalisation. Revue internationale de communication sociale et publique, $3 / 4$. 
Brulois, V. et Charpentier, J-M. (2009, mai). La dimension sociale au cœur de la communication présentée lors du $77^{e}$ Congrès ACFAS, Ottawa, Canada.

Brulois, V. et Viers, J. (2009, juin). Plaidoyer pour une analyse transdisciplinaire de la RSE présentée lors du $4^{e}$ Congrès RIODD, Lille, France.

Courpasson, D. (2011, juin). Des résistants chez les cadres? Cahiers de la communication interne, (28), 10-12.

Dupuy, F. (2011). Lost in Management. La vie quotidienne des entreprises au xxi siècle, Paris : éditions Seuil.

Dupuy, F. (2010, novembre). Entretien : "Il faut sortir de la religion des processus". Liaisons sociales, (116), 6-8.

Enlart, S. (2011, septembre). L'entreprise engagée : cohésion sociale, performances et société. Note d'orientation d'Entreprise \& Personnel, 298.

Ferreras, I. (2007). Critique politique du travail : travailler à l'heure de la société des services, Paris, France : éditions Presses de Sciences Po.

Fleury, C. (2011, juin). Le courage, une force motrice. Cahiers de la communication interne, (28), 21-25.

Fleury, C. (2010). La Fin du courage, Paris : éditions Fayard.

Gomez, P-Y. (2010, 9 mars). De l'importance d'être distant. Le Monde.

Habib, L. (2011). Dossier de presse, La Communication transformative : pour en finir avec les idées vaines [en ligne]http://www.communicationtransformative.fr/d1/2010/09/Dossier_de_presse_Communication_transformativ e.pdf

Hatchuel, A. (2011, 22 novembre). Les épreuves de l'apprenti dirigeant. Le Monde.

Hatchuel, A. (2010, 12 janvier). Dialoguer et critiquer, un travail nécessaire. Le Monde.

Inergie (2009, novembre). Baromètre de la communication interne 2009 [en ligne]

http://www.afci.asso.fr/publication/barometre-de-la-fonction-communicationinterne-2009

di Lampedusa, G. T. (1958). Le Guépard, Paris : éditions Seuil, Paris, 2007 (1 éd. : 1958).

Le Gall, J-M. (2010, 9 mars). Vers une gestion désincarnée des ressources humaines. Le Monde.

Méda, D. (2011, 22 novembre) : Comment s'épanouir au travail ? Le Monde. 
Mintzberg, H. (2009). Misère du management. (entretien avec H.Mintzberg). Revue du Mauss, 1 (33), 247-252.

MispelblomBeyer, F. (2007). Travailler, c'est lutter, Paris : éditions L'Harmattan.

Peretti, J-M. (dir.) (2006). Tous DRH. Les responsabilités Ressources humaines des cadres et dirigeants, Paris : éditions d'Organisation.

Porter, M. et Kramer, M. (2011, janvier-février). CreatingShared Value. Harvard Business Review. [enligne] http://www.waterhealth.com/sites/default/files/Harvard_Buiness_Review_Share d_Value.pdf.

Rozès, S. (2011, juin). La défiance française. Les Cahiers de la communication interne, (28), 17-20.

Segrestin, D. (2003). Pour une sociopolitique du management. Dans J-C. Sardas, D. Giauque, A-M. Guénette(dir.), Comprendre et organiser. Quels apports des sciences humaines et sociales?, Paris : éditions L'Harmattan.

Thuderoz, C. (1997). Sociologie des entreprises, Paris : éditions La Découverte.

Tixier, P-É. (dir.) (2010). Ressources humaines pour sortie de crise, Paris : éditions Sciences Po Les Presses.

Uhalde, M. (2010, décembre). Transformations, individus et... communicants. Regards croisés de chercheurs. Les Cahiers de la communication interne, (27), 27-33.

Zarifian, P. (2010, décembre). La communication dans le travail pour se comprendre et faire société. Les Cahiers de la communication interne, (27), 22-26. 